\title{
Generalized stacking fault energy surfaces and dislocation properties of silicon: A first-principles theoretical study
}

\section{Citation}

Juan, Yu-Min, and Efthimios Kaxiras. 1996. "Generalized Stacking Fault Energy Surfaces and Dislocation Properties of Silicon: A First-Principles Theoretical Study." Philosophical Magazine A 74 (6): 1367-84. https://doi.org/10.1080/01418619608240729.

\section{Permanent link}

http://nrs.harvard.edu/urn-3:HUL.InstRepos:41384067

\section{Terms of Use}

This article was downloaded from Harvard University's DASH repository, and is made available under the terms and conditions applicable to Other Posted Material, as set forth at http:// nrs.harvard.edu/urn-3:HUL.InstRepos:dash.current.terms-of-use\#LAA

\section{Share Your Story}

The Harvard community has made this article openly available. Please share how this access benefits you. Submit a story.

Accessibility 


\title{
Generalized Stacking Fault Energy Surfaces and Dislocation Properties of Silicon: A First-Principles Theoretical Study
}

\author{
Yu-Min Juan and Efthimios Kaxiras \\ Department of Physics and Division of Applied Sciences \\ Harvard University, Cambridge MA 02138
}

June 22, 2018

\begin{abstract}
The generalized stacking fault (GSF) energy surfaces have received considerable attention due to their close relation to the mechanical properties of solids. We present a detailed study of the GSF energy surfaces of silicon within the framework of density functional theory. We have calculated the GSF energy surfaces for the shuffle and glide set of the (111) plane, and that of the (100) plane of silicon, paying particular attention to the effects of the relaxation of atomic coordinates. Based on the calculated GSF energy surfaces and the Peierls-Nabarro model, we obtain estimates for the dislocation profiles, core energies, Peierls energies, and the corresponding stresses for various planar dislocations of silicon.
\end{abstract}

\section{Introduction}

An issue of central importance in materials science is the intrinsic ductility or brittleness of solids. To address this question, one has to consider how a crack 
in the solid will respond to external loading. Since dislocation nucleation at a crack tip will cause the tip to blunt, it is customary to associate this process with ductile behavior. In contrast, brittle behavior is associated with crack propagation without dislocation emission, corresponding to the creation of a sharp crack tip. Therefore, the intrinsic ductility or brittleness of a solid can be determined by comparing the likelihood for dislocation nucleation at the crack tip to Griffith's criterion for cleavage (Griffith (1920)).

Estimating the likelihood of dislocation nucleation at a crack tip under external loading is a non-trivial task. Significant advances have been made by Rice and collaborators recently in modeling dislocation nucleation at a crack tip based on the Peierls stress concept (Rice (1992), Beltz and Rice (1991, 1992), Rice and Beltz (1994), Rice, Beltz, and Sun (1992), Beltz (1992), Sun, Beltz, and Rice (1993)) In that work, an important solid state parameter, the unstable stacking fault energy denoted by $\gamma_{u s}$, was identified as the controlling parameter for dislocation emission at a crack tip under shear loading. One of the attractive features of Rice's theory is that the value of $\gamma_{u s}$ can be obtained from theoretical calculations without any ambiguity: Consider the process by which an infinite crystal is cut in half along a plane, and the upper half is sheared with respect to the lower half by a displacement vector $\vec{f}$ and let $\Phi(\vec{f})$ be the energy per unit area on the slip plane associated with this displacement. The energy surface $\Phi(\vec{f})$ obtained as a function of the generalized displacement vector $\vec{f}$ is called the generalized stacking fault (GSF) energy surface. The GSF energy surface or $\gamma$ surface and its relation to mechanical properties of solids were first considered by Vitek (1966), (1967), (1968), Vitek and Yamaguchi (1973), and Yamaguchi and Vitek (1975). In this context, Rice's unstable stacking energy $\gamma_{u s}$ is the lowest energy barrier in the $\gamma$ surface that has to be surmounted during the shearing process that takes a crystal from an ideal configuration to another equivalent one.

In addition to its relevance to dislocation emission, the GSF energy surface can be used to obtain the restoring force due to the misfit of the lattice near the core 
of a dislocation (see for example, Jòos, Ren, and Duesbery (1994)). By incorporating these restoring forces into the Peierls-Nabarro model (Peierls (1940), Nabarro (1947)), one can calculate dislocation profiles, core energies, Peierls energies, and Peierls stresses for planar dislocations on the corresponding crystal plane. The calculation of dislocation properties within the Peierls-Nabarro model represents a drastic simplification, because it neglects the discreteness of the lattice by treating the problem in a continuum picture. Despite its obvious limitations, this approach provides a useful phenomenological framework for comparison of the structural properties as well as the energetics of various dislocations.

From the above discussion, it is evident that an accurate description of the GSF energy surface is desirable. Empirical methods based on classical interatomic potentials are not sufficiently accurate for this task (Duesbery, Michel, Kaxiras, and Jòos (1991)). Given the significance of GSF energy surfaces, it is important to obtain these values from a first-principles theoretical point of view, i.e. from calculations which are free of adjustable parameters. First-principles calculations based on Density Functional Theory (DFT) (Hohenberg and Kohn (1964), Kohn and Sham (1965)), which are computationally much more demanding than empirical approaches, have been shown to be very successful in predicting the energy differences between different structures of solids. We have performed first-principles DFT calculations for both the shuffle and glide sets of the (111) plane as well as the (100) plane of silicon in the diamond lattice. There are several reasons for choosing these particular planes. These are low index planes of this crystal system, with in-plane dense packing of the atoms and relatively large spacing between planes. Therefore, these planes are natural candidates to be exposed during cleavage. Moreover, the Burgers vectors associated with dislocations on these planes are short compared to other planes, which suggests a smaller dislocation core energy.

The remaining of this paper is organized as follows: Section 2 describes the computational techniques used in our first-principles calculations for GSF energy surfaces. Section 3 contains our results for the GSF energy surfaces of the (111) 
and (100) planes of silicon. The effects due to the relaxation of atomic coordinates are also discussed there. In Section 4 we give a brief review of the Peierls-Nabarro model and then present the dislocation properties obtained within this model from the calculated GSF energy surfaces. We conclude with some remarks on the applicability of Peierls-Nabarro model in Section 5 .

\section{Computational Methods}

The local density approximation (LDA) to the exchange-correlation functional of DFT proposed by Perdew and Zunger (1984) was used for the GSF energy calculations. The valence electron wave functions were expanded in a plane wave basis. The highest kinetic energy of the plane waves included in the basis set is $8 \mathrm{Ry}$. The ionic potential, including the screening by core electrons, was modeled by a nonlocal norm-conserving pseudopotential from Bachelet, Greenside, Barraff, and Schlüter (1982), and the scheme of Kleinman and Bylander (1982) was employed to make the potential separable in Fourier space. The $d$ angular-momentum component was treated as the local part of the potential with the $s$ and $p$ components containing the nonlocal contributions. To simulate the block shearing process, a supercell consisting of 12 atomic planes in the direction perpendicular to the cut was used in these calculations. For the reciprocal space integration, we have used 20 special k-points in the irreducible Brillouin zone, using the scheme of Monkhorst and Pack (1976). The approach of Car and Parrinello (1985), which allows simultaneous relaxation of both the ionic and the electronic degrees of freedom, was employed in the present calculations. The minimum energy was obtained with the steepest descent method. Atoms on the four atomic planes farthest from the plane of the cut were kept frozen to simulate the effects of the bulk. Atomic relaxations are taken into account my minimizing the magnitude of forces calculated from the Hellmann-Feynman theorem. The structures are considered fully relaxed when the magnitude of the forces is smaller than $0.005 \mathrm{Ry} / \mathrm{a} . \mathrm{u}$. 
The unrelaxed GSF energy surface is obtained by simply moving one half of the crystal rigidly with respect to the other half. This GSF energy surface can be altered when atoms on either side of the cut are allowed to respond to shearing forces. The difference in GSF energy surfaces with and without atomic relaxation can be very important, and can even change siginificantly the magnitude of $\gamma_{u s}$ as well as the configuration corresponding to it (see next section).

There is one subtlety that needs to be clarified in relation to the relaxation of ionic coordinates. Within Rice's continuum theory (Rice (1992)) the GSF energy is defined as a function of the displacement on either side of a mathematical cut in the middle of the crystal. In a real crystal, the closest approximation to this displacement is the relative displacement $\vec{f}$ of the two atomic planes immediately adjacent to the cut. When the two blocks on either side of the cut are moved rigidly, $\vec{f}$ is identical to the relative displacement of the centers of the two halves of the crystal. When relaxation is included, special care must be taken in interpreting the proper value of $\vec{f}$ from the in-plane coordinates of the ions. For certain values of $\vec{f}$, where $\Phi(\vec{f})$ is required by symmetry to have an extremum (as in the case of $\left.\gamma_{u s}\right)$, only relaxations that are perpendicular to the plane of the cut are allowed. In these cases, $\vec{f}$ is again identified with the relative displacement of the centers of the two halves of the crystal. When symmetry constraints are absent, atomic relaxations in all directions are allowed. In these cases, the correct value of the relative displacement $\vec{f}$ is obtained from the relaxed in-plane coordinates of atoms on the two planes immediately adjacent to the cut, rather than from the relative displacement of the centers of the two halves of the crystal. Therefore, for a given relative displacement $\vec{f}_{C}$ between the centers of the two halves of the crystal, atomic relaxation not only reduces the energy but also defines the actual displacement $\vec{f}$ (generally $\vec{f} \neq \vec{f}_{C}$ ), which is the value relevant to the GSF energy surface. 


\section{GSF Energies}

We first consider the (111) plane which is the natural cleavage plane of silicon. For this plane of the diamond lattice, there are two distinct ways to cut the crystal: the shuffle set and the glide set. The difference between these two ways is shown in Fig. 1(a). For the shuffle set, the vertical distance between the two adjacent atomic planes immediately above and below the cut is equal to the bond length; only one bond per atom on either side of the cut is broken during the block shearing process. The corresponding interplanar distance for the glide set is only $1 / 3$ of the bond length; three bonds per atom on either side of the cut are broken in the slip along this plane. The calculated GSF energy surfaces for the corresponding cuts in rigid block shearing with all the atoms in the two respective half crystals held fixed during shearing, are shown in Fig. 2(a) and 3(a) respectively. The effects of atomic relaxation on the GSF energy surfaces are shown in Fig. 2(b) and 3(b) for the shuffle and glide sets. Because the distance between the two atomic planes adjacent to the cut is sufficiently large in the shuffle set, the relaxation effects on this energy surface are very small and hardly noticeable on the scale of $\gamma_{u s}$, which is of order $2 \mathrm{eV}$ [compare Fig. 2(a) and 2(b)].

The effect of atomic relaxation is much more pronounced in the glide set. In Fig. 1 we show the atomic positions on a (110) plane, during the shearing on the glide plane along the $<1 \overline{2} 1>$ direction, for unrelaxed structures. Fig. 1 (a) is the original ideal structure. Fig. 11(b) is the structure for the unstable stacking fault configuration. Fig. 1(c) is the stable stacking fault configuration for the glide set. There is essentially no difference between the stable stacking fault structure and the ideal structure, as far as the coordination numbers of the two sets of atoms adjacent to the cut are concerned. For this reason, the energy of the stable stacking fault configuration is very low (see Fig. 3). For displacements beyond the stable stacking fault configuration along the $<1 \overline{2} 1>$ direction, the atoms belonging to the same color (white or black) immediately adjacent to the cut start getting 
very close to each other as can be seen from Fig. 1(d), (e) and (f). Relaxation is restricted by symmetry to the direction perpendicular to the plane of the cut for configurations 1(b), (c) and (e). Especially in Fig. 11(e), the atoms are within a very small distance, almost on top of each other. This kind of arrangement of atoms costs a significant amount of energy due to the strong repulsive interaction between the ionic cores. Configuration 1(e) actually corresponds to the highest energy for the glide cut. The relaxed energy surface on the glide set has the same energy scale as that for the shuffle set. The reason for this dramatic reduction in energy [compare Fig. 3(a) and 3(b)] is that through relaxation the atoms can avoid coming very close to each other during the sliding process.

The results for the (100) plane before and after atomic relaxation of the atomic coordinates are shown in Fig. 4(a) and 4(b) respectively. The origin for the high energy barrier along the $<01 \overline{1}>$ path is similar to the situation for the glide set, where atoms adjacent to the cut come very close to each other. Therefore, the relaxation of the atomic coordinates is expected to reduce the energy barrier along this direction significantly. As is shown in Fig. 4, the topology of the energy surface is different after atoms are allowed to relax. One point deserves further attention: the $<01 \overline{1}>$ path, which is the path containing the highest energy barrier before relaxation, has become the energetically favorable path for the gliding process on the (100) plane after taking into account the effects of atomic relaxation.

The calculated values of $\gamma_{u s}$ both before and after the relaxation of atomic coordinates for the (111) and (100) planes of silicon are summarized in Table I. The expressions that give the relaxed GSF energy surfaces, fitted by sinusoidal expansions, are given in the Appendix.

Based on Rice's theory, a direct comparison of the values of $\gamma_{u s}$ indicates that it is energetically more favorable to nucleate dislocations on the shuffle set of the (111) plane under zero temperature and zero pressure conditions. The changes in the energy surface due to atomic relaxation will also affect the estimates of the free energy. Following Kaxiras and Duesbery (1993), we define the free energy per 
unit area associated with a particular slip process as:

$$
F=\gamma_{u s}-T \frac{S}{A}-P \frac{\Delta V}{A}
$$

where $S$ is the entropy and $\Delta V$ the volume relaxation at the saddle point. $\Delta V$ is obtained by minimizing the energy of the saddle point configuration with respect to the magnitude of the slab thickness. We use Vineyard's transition state theory (Vineyard (1957)) as outlined by Kaxiras and Duesbery (1993), to estimate the entropy $S$ for the relaxed energy surface. The condition that the preferred slip plane changes from shuffle to glide is then given by $F^{\text {glide }}=F^{\text {shuffle }}$. The $(P, T)$ values that satisfy this condition are shown in Fig. 5. As anticipated in the work of Kaxiras and Duesbery (1993), the larger reduction in the energy associated with relaxation of the glide cut results in an overall shift of the $F^{\text {glide }}=F^{\text {shuffle }}$ line towards higher pressure and lower temperature, compared to the unrelaxed results. For example, the stress needed to change from the shuffle to the glide set at room temperature $\left(300^{0} \mathrm{~K}\right)$ corresponds to an external pressure of only $15 \mathrm{kbar}$ as calculated from the results with the relaxed energy surface, while a pressure as high as 53 kbar would be predicted if the unrelaxed energy surface were used.

However, even the results obtained from the relaxed energy surface calculations should not be taken literally for the following reasons: (1) Other factors (such as the electronic degrees of freedom), which are neglected in the present entropy calculation, could make a significant contribution to the free energy. (2) The comparison of free energies is based on Rice's theory in which the energy associated with the creation of surface during dislocation emission is neglected; further investigations are needed to get an estimate of such effects (see for instance $\mathrm{Xu}$, Argon and Ortiz (1995)). A detailed discussion on surface effects in dislocation nucleation for silicon will be published elsewhere (Juan, Kaxiras, and Sun (1995)). (3) Effects related to reconstruction of the dislocation core are also neglected in this theoretical framework. For a stiff covalent material, core reconstruction effects are very important (see Bulatov, Yip and Argon (1995)), and are expected to change 
the values of $\gamma_{u s}$. All these approximations may affect the exact numbers for the transition from shuffle to glide dominance, even though we expect the qualitative picture to remain the same.

\section{Peierls-Nabarro Model for Planar Dislocation}

By combining our results for the GSF energy surfaces with the Peierls-Nabarro model (Peierls (1940), Nabarro (1947)), we can further analyze the properties of dislocations on these planes within continuum elastic theory. We give here a brief description of the Peierls-Nabarro model which is based on the premise that a dislocation can be thought of as a continuous distribution of infinitesimal crystal misfits on the glide plane (Kroupa and Lejček (1972), Hirth and Lothe (1982), Jòos et al. (1994)). In the following we treat the distortion due to the dislocation as a scalar function of position $f(x)$ for simplicity, although the true vectorial character

of $\vec{f}$ should be considered in a general treatment. For each point on the glide plane at a distance $x^{\prime}$ from the dislocation line, there is a corresponding infinitesimal Burgers vector $d f^{\prime}=d f /\left.d x\right|_{x^{\prime}} d x^{\prime}$, where $d f / d x$ is defined to be the dislocation density $\rho(x)$ and $f(x)$ is the disregistry at the point $x$, satisfying the condition:

$$
\int_{-\infty}^{+\infty} \rho\left(x^{\prime}\right) d x^{\prime}=\int_{-\infty}^{+\infty} \frac{d f\left(x^{\prime}\right)}{d x^{\prime}} d x^{\prime}=b
$$

with $b$ the Burgers vector of the dislocation. From the elastic model, the stress due to a dislocation along the direction of the Burgers vector on the slip plane is given by

$$
\sigma_{b n}=\frac{K}{2 \pi} \frac{b}{x}
$$

where $x$ is the distance between the dislocation and the point where the stress is evaluated, and $n$ is the normal to the glide plane. The constant $K$ depends on the elastic properties of the crystal and the dislocation type. Its value for an isotropic solid is given by Jòos et al. (1994):

$$
K=\mu\left[\frac{\sin ^{2}(\theta)}{(1-\nu)}+\cos ^{2}(\theta)\right]
$$


where $\mu$ and $\nu$ are the shear modulus and Poisson's ratio respectively, and $\theta$ is the angle between the dislocation line and the Burgers vector. Therefore, the stress due to the existence of the dislocation for a point $x$ on the glide plane is:

$$
\frac{K}{2 \pi} \int_{-\infty}^{+\infty} \frac{\rho\left(x^{\prime}\right)}{x-x^{\prime}} d x^{\prime}
$$

Meanwhile, there is another stress which is due to the local disregistry of the crystal and tends to restore the crystal. This crystal restoring stress is a periodic function of the displacement and can be represented as $F_{b}(f)$. Equilibrium is attained when the stresses due to the two terms are in balance with each other as expressed in the following equation, known as the Peierls-Nabarro equation,

$$
\frac{K}{2 \pi} \int_{-\infty}^{+\infty} \frac{\rho\left(x^{\prime}\right)}{x-x^{\prime}} d x^{\prime}=F_{b}(f(x))
$$

The dislocation profile $f(x)$ and the dislocation density $\rho(x)=d f / d x$ can be determined by solving this equation with the normalization condition $\int_{-\infty}^{+\infty} \rho(x) d x=b$. In the original Peierls-Nabarro model, a simple sinusoidal form is assumed for the restoring stress,

$$
F_{b}(f(x))=F_{\max } \sin \left(\frac{2 \pi f(x)}{b}\right),
$$

with $F_{\max }$ the maximum stress. This assumption leads to the analytic solution

$$
f(x)=\frac{b}{\pi} \tan ^{-1} \frac{x}{\zeta}+\frac{b}{2} .
$$

The parameter $\zeta=K b / 4 \pi F_{\max }$ can be viewed as the width of the dislocation within this model, since the value of the dislocation density $\rho$ at this point is exactly one half its value at $x=0$. Within the GSF approach the crystal restoring force $F_{b}(f)$ can be obtained by simply taking the derivative of the GSF energy surface $\gamma(f)$ with respect to the lattice distortion $f$ (Kroupa and Lejček (1972), Vitek and Yamaguchi (1973), Yamaguchi and Vitek (1975)). For a general functional form of $F$, there is no analytic solution for $f(x)$. We follow the procedure of Joós et al. (1994) to solve the Peierls-Nabarro equation numerically. This is done by expressing the disregistry vector $f(x)$ as a series

$$
f(x)=\frac{b}{\pi} \sum_{i=1}^{n} \alpha_{i} \tan ^{-1} \frac{x-x_{i}}{\zeta_{i}}+\frac{b}{2},
$$


with the parameters $\alpha_{i}, \zeta_{i}, x_{i}$ to be determined, subject to the normalization condition

$$
\sum_{i=1}^{n} \alpha_{i}=1
$$

since the total Burgers vector should be equal to $b$. By substituting the above displacement formula into the two sides of the Peierls-Nabarro equation, we get the forces $F\left(\alpha_{i}, \zeta_{i}, x_{i}, x\right)$ and $F^{\prime}\left(\alpha_{i}, \zeta_{i}, x_{i}, x\right)$ corresponding to the contributions from dislocation distribution and crystal distortion respectively. The difference between these two forces $\left|F\left(\alpha_{i}, \zeta_{i}, x_{i}, x\right)-F^{\prime}\left(\alpha_{i}, \zeta_{i}, x_{i}, x\right)\right|$ is then minimized by varying the parameters $\alpha_{i}, \zeta_{i}$ and $x_{i}$ so that the Peierls-Nabarro equation is satisfied numerically. We find that a numerical solution is feasible by retaining only three terms in Eq. (9).

We display in Fig. 6 the GSF energies and forces obtained from our firstprinciples calculations with the full relaxation of the atomic coordinates, along the directions which are relevant to the dislocations we will consider. For the case of the $<1 \overline{2} 1>$ direction on the glide plane, only the portion ranging from the ideal structure to the stacking fault configuration is shown, since a partial dislocation will be formed along this direction. One feature of these curves deserves further comment: not all the restoring forces can be well described by a simple sinusoidal from (see also the recent work of Xu, Argon and Ortiz (1995)). Especially in the case of the (100) plane, the force curve is very flat when the displacement is approximately one half of the full Burgers vector. The significance of this deviation will be discussed in detail later, when we consider the profiles of various dislocations.

The disregistry vector for full dislocations on the shuffle set of the (111) plane, the glide set of the (111) plane, and the (100) plane are displayed in Fig. 7 (a), (b), and (c) respectively. We note that the dislocations with Burgers vector along the $<10 \overline{1}>$ direction on the glide plane (glide- $60^{0}$ and glide-screw) are more concentrated compared to the other dislocations on the (111) plane (this effect will become more obvious when we compare the dislocation density). This difference in the distribution density can be understood by considering the magnitude of 
the crystal restoring force for these dislocations. A qualitative estimate can be obtained by considering the half width of the dislocation $\zeta$ within the classical model, which is inversely proportional to the magnitude of the crystal restoring force. This force is largest in the $<10 \overline{1}>$ direction of the glide plane, as is evident by comparing Figures 2, 3 and 4 .

In Fig. 8 we show representative dislocation densities for the planes we have considered, including (a) the $60^{0}$ dislocation on the shuffle set, (b) the $60^{0}$ dislocation on the glide set, and (c) the $90^{\circ}$ dislocation on the (100) plane. We have displayed the results obtained from both the relaxed (upper panel) and the unrelaxed GSF energy surfaces (lower panel) to examine the effects due to the relaxation of the atomic coordinates. For comparison we have also displayed the results from the classical solution (dashed curves), obtained by assuming a sinusoidal form for the crystal restoring force with its maximum equal to the value obtained from the first-principles calculations. For the case of the $60^{0}$ dislocation on the shuffle set, it is apparent that the use of the relaxed GSF energy surface does not cause significant change on the dislocation profile both qualitatively and quantitatively. This is a consequence of the fact that atomic relaxation does not change the GSF energies along this direction in any significant way. For the $60^{0}$ dislocation on the glide set, the use of the relaxed energy surface makes the dislocation profile considerably smoother and wider but the general shape remains unchanged. This is due to the fact that the relaxation reduces the magnitude of the crystal restoring force but does not change its functional form significantly. For the case of the $90^{\circ}$ dislocation on the (100) plane, the dislocation profile is changed qualitatively with the use of the relaxed energy surface. This reflects the fact that the crystal restoring force for this plane is significantly different from the sinusoidal function as we mentioned above. The double peak indicates the dissociation of the full dislocation into two fractional dislocations (Vitek and Kroupa (1969)). A brief summary of the obtained dislocation properties is given in Table II.

Another useful quantity to consider is the energy barrier associated with dislo- 
cation motion. Within the Peierls-Nabarro model, this energy barrier is called the Peierls energy and is defined as the amplitude of the variation of the misfit energy on the glide plane as the position of the dislocation line moves. With the obtained dislocation profiles, we can calculate the misfit energy across the glide plane as a function of the position of the dislocation line $u$ following the definition given by Vitek and Yamaguchi (1973) and Jóos et al. (1994). To be consistent with the Peierls-Nabarro model, where the discreteness of the crystal is taken into account at the glide plane, the misfit energy $W(u)$ is defined as the sum of all the misfit energies between pairs of atom rows, obtained from the GSF energy at the local disregistry:

$$
W(u)=\sum_{m=-\infty}^{+\infty} \gamma\left(f\left(m a^{\prime}-u\right)\right) a^{\prime},
$$

where $a^{\prime}$ is the distance between adjacent atomic planes in the direction perpendicular to the dislocation line. This expression meets two important requirements: First, it has the correct period $a^{\prime}$ of planes in the crystal,

$$
W\left(u+a^{\prime}\right)=W(u)
$$

Second, in the limit of a very narrow dislocation, it reproduces the correct maximum $\gamma_{u s} a^{\prime}$. The other quantity which might be of interest is $W\left(a^{\prime} / 2\right)$ where the minimum of the misfit energy function occurs. Since this quantity measures the nonelastic part of the energy of the dislocation, it provides a qualitative estimate of the core energy. The stress associated with the energy function $W(u)$ can then be defined as:

$$
\sigma(u)=\left.\frac{1}{a^{\prime}} \frac{d W\left(u^{\prime}\right)}{d u^{\prime}}\right|_{u^{\prime}=u}
$$

The maximum of this stress function is the Peierls stress, needed to move the dislocation. The values of the energy function $W(u)$ and the stress function $\sigma(u)$ for the (111) plane are shown in Fig. 9 (a) and (b). It is obvious that the dislocation with Burgers vector along the $<10 \overline{1}>$ direction on the glide set (labeled Glide- $60^{0}$ in Fig. 9 (b)) has larger Peierls energy and Peierls stress. This is expected since we know from our GSF energy surface calculations that the energy barrier along 
that direction is higher than those along the other two vectors. Fig. 9(c) is the energy and stress function for the case of the (100) plane. The different behavior between the $90^{\circ}$ and the screw dislocations on the (100) plane is primarily due to the difference in the values of the constant $K$. The Peierls energy, stress, and core energy of various dislocations are summarized in Table III. For the (111) plane, it appears that the dislocations belonging to the shuffle set are the easiest to move under zero temperature and zero pressure conditions. However, as we discussed earlier, including the effects of temperature, pressure, surface creation and core reconstruction can change the picture. Finally, we would like to mention that there is experimental evidence that on the glide set the $90^{\circ}$ partial is more mobile than the $30^{\circ}$ partial (Wessel and Alexander (1977), Alexander, Gottschalk, and Kisielowski-Kemmerich (1985), Grosbras, Demenet, Garem, and Desoyer (1984), Demenet, Grosbras, Garem, and Desoyer (1989)), which is consistent with the results of our calculations, namely $\sigma(u)$ is lower for the glide- $90^{0}(\mathrm{p})$ than the glide$30^{0}(\mathrm{p})$ dislocation, see Fig. 9(b).

\section{Summary}

In summary, we have performed first-principles calculations to obtain the GSF energy surfaces for both the shuffle and glide sets of the (111) plane, as well as the (100) plane of silicon. We showed that for the glide set and the (100) plane, the

effects on the GSF energy surfaces due to the relaxation of the atomic coordinates are significant. The unstable stacking fault energies $\gamma_{u s}$ for these planes were determined from our calculations. By combining these values with Rice's criterion for dislocation nucleation, the shuffle set appears to be favored for dislocation emission under zero temperature and zero pressure conditions. A qualitative account of entropy effects was attempted, based on Vineyard's transition state theory. By comparing the free energies of the shuffle and glide sets, we find that either set can dominate under different thermodynamical conditions. Quantitative free-energy 
comparisons should also take into account additional entropy effects, and the effects of surface creation and core reconstruction.

The GSF energy surfaces were then combined with the Peierls-Nabarro model to investigate the dislocation properties of silicon. The crystal restoring forces are obtained directly from our first-principles GSF energy surfaces. We demonstrated the importance of using the relaxed energy surface for the calculation of these properties. The differences between dislocation profiles obtained with relaxed GSF and unrelaxed GSF energy surface are significant in certain cases. The Peierls energy and Peierls stress were also calculated within the framework of the PeierlsNabarro model.

We would like to conclude with a brief discussion on the approximations involved in the Peierls-Nabarro model: (1) The dislocation is assumed to be planar within this model. We expect that the errors introduced with this approximation for a stiff material like silicon should be minimal. (2) The response from the crystal is treated within elastic theory, which may not apply for a very narrow core situation, where non-elastic effects are expected to be important. (3) The Peierls stress concept assumes that the only relevant quantity in determining the stress is the local disregistry, which is true only when the dislocation density is very smooth. (4) The dislocation line is assumed to move as a rigid object, which is unlikely (see e.g. the recent work of Bulatov, Yip and Argon (1995) on this issue). Accordingly, we suggest that these results should not be taken literally. Rather, our investigation provides a qualitative comparative discussion of dislocation properties in silicon, that can serve as guide to more detailed studies.

\section{Acknowledgement}

We are indebted to J.R. Rice and his coworkers Y. Sun and G. Beltz for useful discussions and insight on GSF theory. We acknowledge useful discussions with M.S. Duesbery, B. Jòos, A. Argon and S. Yip on dislocation properties. We are 
particularly thankful to $\mathrm{V}$. Bulatov for a critical reading of the manuscript and many useful suggestions.

This work was supported in part by ONR grant \#N00014-92-J-1960 and in part by Harvard's Materials Research Science and Engineering Center, which is funded through NSF. The calculations were performed at the Department of Defense NAVOCEANO and CEWES computational facilities. 


\section{Appendix}

We used sinusoidal expansions to fit the calculated GSF energy surfaces in order to facilitate the computation of dislocation properties. The expansions were chosen so that they satisfy the underlying translational symmetries of the lattice. We have checked the numerical values and found that the numbers obtained from the fittng formula reflect the underlying rotational symmetry with sufficient accuracy. Specifically, we use the following expression:

$$
\begin{aligned}
\gamma(x, y)= & \sum_{n, m} A_{n m} \cos \left(\frac{2 \pi n}{a_{1}} x\right) \cos \left(\frac{2 \pi m}{a_{2}} y\right)+B_{n m} \cos \left(\frac{2 \pi n}{a_{1}} x\right) \sin \left(\frac{2 \pi m}{a_{2}} y\right) \\
& +C_{n m} \sin \left(\frac{2 \pi n}{a_{1}} x\right) \sin \left(\frac{2 \pi m}{a_{2}} y\right)
\end{aligned}
$$

where $a_{1}$ and $a_{2}$ are the repeat distances in the $(x, y)$ plane. In terms of the lattice constant $a_{0}$ of bulk Si, these are given by $a_{1}=a_{0} / \sqrt{2}, a_{2}=a_{0} \times \sqrt{3 / 2}$ for the (111) plane cuts (both shuffle and glide), and $a_{1}=a_{2}=a_{0} / \sqrt{2}$ for the (100) plane. The $(x, y)$ directions in the (111) plane cuts correspond to the $<10 \overline{1}>$ and $<1 \overline{2} 1>$ crystallographic directions, whereas in the (100) plane cut they correspond to the $<011>$ and $<01 \overline{1}>$ crystallographic directions. The coefficients of the terms retained in the above expansion are given in Table IV.

\section{References}

Alexander H., Gottschalk H., and Kisielowski-Kemmerich C., (1985) in Dislocations in Solids, edited by H. Suzuki, T.Minomoya, K. Sumino, and S. Takeuchi (University of Tokyo Press, Tokyo).

Bachelet G., Greenside H., Barraff G., and Schlüter M., (1981) Phys. Rev. 24, 4745.

Beltz G.E., (1992) Ph.D Thesis, Division of Applied Sciences, Harvard Univer- 
sity, Cambridge, MA.

Beltz G.E. and Rice J.R., (1991) in Modeling the Deformation of Crystalline Solids: Physical Theory, Applications, and Experimental Comparisons, edited by T.C. Lowe, A.D. Rollett, P.S. Follansbee and G.S. Dehn, (TMS Minerals, Metals and Materials Society, Warrendale).

Beltz G.E. and Rice J.R., (1992) Acta Metall. 40 S321.

Bulatov V.V., Yip S., and Argon, A.S. (1995) Phil. Mag. A 72, 453.

Car R. and Parrinello M., (1985) Phys. Rev. Lett. 55, 2471.

Demenet J.L., Grosbras P., Garem H., and Desoyer J.C., (1989) Phil. Mag. A 59, 501

Duesbery M.S., Michel D.J., Kaxiras E., and Jòos B., (1991) Mat. Res. Soc. Symp. Proc. Vol. 209, p. 125.

Griffith A.A., (1920) Phil. Trans. R. Soc. A 184, 181.

Grosbras P., Demenet J.L., Garem H., and Desoyer J.C., (1984) Phys. Status Solidi A 84, 481.

Hirth J.P. and Lothe J., (1982) Theory of Dislocations, 2nd ed. (Wiley, New York).

Hohenberg P. and Kohn W., (1964) Phys. Rev. 136, B864.

Jòos B., Ren Q., and Duesbery M.S., (1994) Phys. Rev. B 50, 5890.

Juan Y.M, Kaxiras E. and Sun Y., (1995) Phil. Mag. Lett. (to be published).

Kaxiras E. and Duesbery M.S., (1993) Phys. Rev. Lett. 70, 3752.

Kleinman L. and Bylander D.M., (1982) Phys. Rev. Lett. 48, 1425.

Kohn W. and Sham L., (1965) Phys. Rev. 140, A1133.

Kroupa F. and Lejček L., (1972) Czech. J. Phys. Rev. B. 22, 813.

Louchet F. and Thibault-Desseaux J., (1987) Rev. Phys. Appl. 22, 207.

Monkhorst H.J. and Pack J.D., (1976) Phys. Rev. B. 13, 5188.

Nabarro F.R.N., (1947) Proc. Phys. Soc. London 59, 256.

Peierls R., (1940) Proc. Phys. Soc. London 52, 34.

Perdew J. and Zunger A., (1984) Phys. Rev. B 23, 5048. 
Rice, J.R., (1992) J. Mech. Phys. Solids, 40, 239.

Rice J.R. and Beltz G.E., (1994) J. Mech. Phys. Solids, 42, 333.

Rice J.R., Beltz G.E., and Sun Y., (1992) in Topics in Fracture and Fatigue, edited by A.S. Argon, (Springer, Berlin).

Sun Y., Beltz G.E., and Rice J.R., (1993) Mater. Sci. Engng A170 67.

Vineyard G.H., (1957) J. Phys. Chem. Solids 3, 121.

Vitek V., (1966) Phys. Stat. Sol. 18, 683.

Vitek V., (1967) Phys. Stat. Sol. 22, 453.

Vitek V., (1968) Phil. Mag. 18, 773.

Vitek V. and and Kroupa F., Phil. Mag. 19, 265.

Vitek V. and Yamaguchi M., (1973) J. Phys. F 3, 537.

Wessel K. and Alexander H., (1977) Phil. Mag. 35, 1523.

Yamaguchi M. and Vitek V., (1975) J. Phys. F 5, 11.

Xu G., Argon A.S. and Ortiz M. (1995), Phil. Mag. A 72, 415. 


\section{Table I}

\begin{tabular}{||c|c|c|c|c||}
\hline \multirow{2}{*}{} & \multicolumn{2}{|c|}{ No Relaxation } & \multicolumn{2}{c||}{$\begin{array}{c}\text { Atomic Relaxation } \\
\text { at ideal volume }\end{array}$} \\
\hline \hline & location & $\gamma_{u s}\left(\mathrm{~J} / \mathrm{m}^{2}\right)$ & location & $\gamma_{\text {us }}\left(\mathrm{J} / \mathrm{m}^{2}\right)$ \\
\hline \hline$(111)$-Shuffle & $\frac{1}{4}[10 \overline{1}]$ & 1.84 & $\frac{1}{4}[10 \overline{1}]$ & 1.81 \\
\hline$(111)$-Glide & $\frac{1}{12}[1 \overline{2} 1]$ & 2.51 & $\frac{1}{12}[1 \overline{2} 1]$ & 2.02 \\
\hline$(100)$ & $\frac{1}{4}[011]$ & 2.97 & $\frac{1}{4}[0 \overline{1} 1]$ & 2.15 \\
\hline \hline
\end{tabular}

TABLE I: The unstable stacking fault energy, $\gamma_{u s}$, obtained for the shuffle set and glide set of the (111) plane, as well as the (100) plane of silicon. The location indicates the position of relative displacement $\vec{f}$ where the $\gamma_{u s}$ occurs.

\section{Table II}

\begin{tabular}{||c|c|c|c|c|c|c|c|c||}
\hline \hline \multirow{2}{*}{ dislocation } & \multicolumn{2}{|c|}{ Shuffle } & \multicolumn{2}{|c|}{ Glide } & \multicolumn{2}{c|}{ Glide partial } & \multicolumn{2}{|c|}{$(100)$} \\
\cline { 2 - 10 } & $60^{0}$ & Screw & $60^{0}$ & Screw & $30^{0}$ & $90^{0}$ & $90^{0}$ & Screw \\
\hline \hline$K\left(10^{11} \frac{d y n e}{c m^{2}}\right)$ & 8.02 & 6.37 & 8.02 & 6.37 & 6.92 & 8.58 & 9.04 & 6.375 \\
\hline$\tau_{\text {max }}\left(10^{11} \frac{d y n e}{c m^{2}}\right)$ & 1.49 & 1.49 & 4.29 & 4.29 & 2.78 & 2.78 & 2.06 & 2.06 \\
\hline$\zeta(\AA)$ & 1.08 & 0.92 & 0.46 & 0.37 & 0.77 & 0.92 & 2.15 & 1.54 \\
\hline $\mathrm{b}(\AA)$ & 3.84 & 3.84 & 3.84 & 3.84 & 2.22 & 2.22 & 3.84 & 3.84 \\
\hline \hline
\end{tabular}

Table II: Quantities related to the properties of dislocations for the shuffle and glide sets on the (111) plane, as well as the (100) plane of silicon. The meaning of $K$ is given in Eq. (4) $), \tau_{\max }$ is the maximum value of $d \gamma_{u s}(\vec{f}) / d \vec{f}$ for the directions we are interested in, $\zeta$ is the half width of the dislocation, and $b$ is the Burgers vector. 
Table III

\begin{tabular}{||c|c|c|c|c|c|c|c|c||}
\hline \hline \multirow{2}{*}{ dislocation } & \multicolumn{2}{|c|}{ Shuffle } & \multicolumn{2}{c|}{ Glide } & \multicolumn{2}{c||}{ Glide partial } & \multicolumn{2}{c||}{$(100)$} \\
\cline { 2 - 9 } & $60^{0}$ & Screw & $60^{0}$ & Screw & $30^{0}$ & $90^{0}$ & $90^{0}$ & Screw \\
\hline \hline$W_{p}(e V \AA)$ & 0.149 & 0.183 & 0.842 & 0.898 & 0.287 & 0.246 & 0.112 & 0.296 \\
\hline$\sigma_{p}\left(e V \AA^{3}\right)$ & 0.046 & 0.062 & 0.399 & 0.504 & 0.176 & 0.139 & 0.032 & 0.081 \\
\hline$W\left(a^{\prime} / 2\right)$ & 0.467 & 0.338 & 0.345 & 0.238 & 0.174 & 0.246 & 0.549 & 0.274 \\
\hline \hline
\end{tabular}

Table III: The Peierls energy $W_{p}$, Peierls stress $\sigma_{p}$, and the estimated core energy $W\left(a^{\prime} / 2\right)$ obtained from the Peierls-Nabarro model for various dislocations on the (111) and (100) planes of silicon.

\begin{tabular}{||l||r|r|r||r|r|r||r||}
\multicolumn{7}{c||}{ Table IV } \\
\hline \hline & \multicolumn{3}{c||}{$(111)$-shuffle } & \multicolumn{3}{c||}{$(111)$-glide } & $(100)$ \\
\hline \hline$(\mathrm{n}, \mathrm{m})$ & $A_{n m}$ & $B_{n m}$ & $C_{n m}$ & $A_{n m}$ & $B_{n m}$ & $C_{n m}$ & $A_{n m}$ \\
\hline \hline$(0,0)$ & 0.9789 & & & 2.2683 & & & 1.8463 \\
\hline$(0,1)$ & & & & & & & -0.6728 \\
\hline$(0,2)$ & -0.3857 & & 0.0625 & -0.7059 & & 0.7024 & \\
\hline$(0,4)$ & 0.0180 & & -0.0200 & 0.2570 & & -0.3473 & \\
\hline$(1,0)$ & & & & & & & -0.8443 \\
\hline$(1,1)$ & -0.7714 & -0.1231 & & -1.3609 & -1.8219 & & -0.2670 \\
\hline$(1,2)$ & & & & & & & -0.1493 \\
\hline$(1,3)$ & 0.0950 & -0.0010 & & -0.6291 & 0.1218 & & \\
\hline$(2,0)$ & 0.0470 & & & -0.0126 & & & 0.1471 \\
\hline$(2,1)$ & & & & & & & 0.0087 \\
\hline$(2,2)$ & 0.0350 & 0.0390 & & 0.4412 & 0.0890 & & -0.0485 \\
\hline$(2,4)$ & & & & -0.2579 & 0.2698 & & \\
\hline$(3,1)$ & -0.0180 & -0.0070 & & & & & \\
\hline \hline
\end{tabular}

Table IV: The non-zero coefficients for the expansion of the $\gamma$ surface in terms of sinusoidal functions for the shuffle and glide (111) cuts, and for the (100) cut. 
Figure 1: The atomic structures involved in the shearing process, in which one half of the diamond crystal is sheared rigidly with respect to the other half along the $<1 \overline{2} 1>$ direction on the glide set of the (111) plane of silicon. Both the glide $(\mathrm{G})$ and shuffle $(\mathrm{S})$ cuts are indicated in part (a) by dashed lines. The two different shadings of the atoms represent two different (110) atomic planes. (a) the ideal structure. (b) the unstable stacking fault configuration. (c) the stacking fault configuration. (d), (e), and (f) are intermediate geometries, when the upper half is sheared further along the $<1 \overline{2} 1>$ direction. (e) is the geometry with the highest energy.

Figure 2: The GSF energy surface: (a) before and (b) after relaxation, for the shuffle set of the (111) plane.

Figure 3: The GSF energy surface: (a) before and (b) after relaxation, for the glide set of the (111) plane.

Figure 4: The GSF energy surface: (a) before and (b) after relaxation, for the (100) plane.

Figure 5: The shuffle set / glide set transition phase diagram on the $(P, T)$ plane. Results including atomic relaxation (solid line) and before relaxation (dashed line) are shown for comparison. For $(P, T)$ values below (above) the line, the glide (shuffle) has lower unstable stacking free energy.

Figure 6: The fully relaxed GSF energy curve and the corresponding stress for the relevant directions on the shuffle, glide and (100) planes of silicon.

Figure 7: (a) The disregistry vector obtained for dislocations on the shuffle set of the (111) plane; (b) the disregistry vector for dislocations on the glide set of the (111) plane; (c) the disregistry vector for dislocations on the (100) plane. 
Figure 8: The dislocation density obtained from our calculations for (a) the $60^{0}$ dislocation on the shuffle set, (b) the $60^{\circ}$ dislocation on the glide set, and (c) the $90^{0}$ dislocation on the (100) plane. Results from both relaxed (upper panel) and unrelaxed (lower panel) GSF energy surfaces are displayed for comparison. The dashed curves are the classical solutions (see text).

Figure 9: (a) The energy function $W(u)$ with respect to the displacement vector $u$ (upper panel) and the corresponding stress $\sigma(u)$ as a function of $u$ (lower panel) for dislocations belonging to shuffle set. (b) Same as in (a), for dislocations on the glide set. (c) Same as in (a) for dislocations on the (100) plane. 